Referencia para citar este artículo: Gaeta, M. L., Cavazos, J. \& Cabrera, M. del R. L. (2017). Habilidades autorregulatorias e higiene bucal infantil con el apoyo de los padres. Revista Latinoamericana de Ciencias Sociales, Niñez y Juventud, 15 (2), pp. 965-978. DOI:10.11600/1692715x.1521109022016

\title{
Habilidades autorregulatorias e higiene bucal infantil con el apoyo de los padres*
}

\author{
MaRTha LETICIA GAETA ** \\ Profesora-investigadora Universidad Popular Autónoma del Estado de Puebla, México. \\ JUDITH CAVAZOS \\ Profesora-investigadora Universidad Popular Autónoma del Estado de Puebla, México.
}

Ma. DEL ROSARIO L. CABRERA****

Directora Clínica Universidad Popular Autónoma del Estado de Puebla, México.

\begin{abstract}
Artículo recibido en febrero 9 de 2016; artículo aceptado en junio 20 de 2016 (Eds.)
\end{abstract}
- Resumen (descriptivo): en este artículo se analizan diferentes variables que inciden en la conducta de auto-cuidado de los pacientes pediátricos que asisten a consulta médica, así como en la de sus padres, para una buena salud oral como son: las creencias de autoeficacia, la intención y el control del comportamiento. En el contexto mexicano, participaron 43 pacientes pediátricos, entre 6 y 13 años de edad, y 36 padres de familia, entre 21 y 47 años de edad, que acompañaban a sus hijos a recibir tratamiento dental. Los resultados muestran que los pacientes pediátricos presentan mayor autoeficacia que su progenitor encuestado; en cambio, los padres presentan mejores respuestas en intención de comportamiento y hábitos de higiene bucal. El control de la acción no presenta diferencias significativas entre los pacientes pediátricos y sus padres.

Palabras clave: autogestión, comportamiento, desarrollo de habilidades, salud bucal(Vocabulario Controlado Iresie).

\section{Self-regulatory skills and child oral hygiene with support from parents}

- Abstract (descriptive): In this article the authors analyze different variables that influence the self-care behavior of pediatric patients attending medical consultations, as well as the behavior of their parents, in relation to achieving good oral health. These variables include: self-belief, behavioral intention and control. In this study conducted in Mexico, participants included 43 pediatric patients aged between 6 and 13 years old, and 36 parents aged between 21 and 47 years of age who accompanied their children to receive dental treatment. The results show that pediatric patients have

\footnotetext{
* Este artículo corto se desarrolló a partir de la investigación en curso denominada "Educación y salud infantil: promoción de habilidades autorregulatorias para el cuidado de los dientes". Financiación aprobada por la Dirección de Investigación de la Universidad Popular Autónoma del Estado de Puebla, el 2 de octubre de 2014; No. de proyecto 133. Área de conocimiento: Ciencias Sociales. Sub-área: Educación.

** Doctora en Psicología y Aprendizaje por la Universidad de Zaragoza, España. Maestra en Psicología por la Universidad de las Américas Puebla, México. Profesora Investigadora del Doctorado en Pedagogía, Universidad Popular Autónoma del Estado de Puebla. Orcid: 0000-0003-1710-217X. Índice H5: 4. Correo electrónico: marthaleticia.gaeta@upaep.mx

*** Doctora en Dirección y Mercadotecnia y Maestra en Mercadotecnia por la Universidad Popular Autónoma del Estado de Puebla, México. Profesora Investigadora del Doctorado en Mercadotecnia, Universidad Popular Autónoma del Estado de Puebla. Orcid: 0000-0001-6538-9553. Índice H5: 5. Correo electrónico: judith.cavazos@upaep.mx

**** Maestra en Desarrollo Humano y Educativo y Cirujano Dentista por la Universidad Popular Autónoma del Estado de Puebla, México. Directora de la Clínica de Odontología, Universidad Popular Autónoma del Estado de Puebla. Orcid: 0000-0002-0947-8364. Correo electrónico: mariadelrosario.cabrera@upaep.mx
} 
higher self-belief than their parents. However, parents have better behavioral intentions and oral hygiene habits than their children. There were no significant differences between pediatric patients and their parents in action control.

Key Words: Self-management, behavior, skills development, oral health

\section{Habilidades de auto-regulação e higiene bucal das crianças com o apoio dos pais}

Resumo (descritivo): Neste artigo se analisam diferentes variáveis que influenciam o comportamento de auto-atendimento de pacientes pediátricos que frequentam consultas médicas, bem como seus pais, para uma boa saúde bucal como são: as crenças de autoeficácia, a intenção e o controle comportamental. No contexto mexicano, participaram 43 pacientes pediátricos entre $6 e$ 13 anos de idade, e 36 pais entre 21 e 47 anos de idade, que acompanharam seus filhos para receber tratamento. Os resultados mostram que os pacientes pediátricos tem maior autoeficácia do que seu progenitor correspondente. Os pais têm melhores respostas na intenção comportamental e hábitos de higiene bucal. O controle da ação não apresenta diferenças significativas entre pacientes pediátricos e seus pais.

Palavras Chave: auto-gestão, comportamento, desenvolvimento de habilidades, saúde oral.

-Introducción. -1. Revisión de la literatura. -2. Metodología. -3. Análisis de resultados. -4. Discusión. -5. Conclusiones. -Lista de referencias.

\section{Introducción}

La caries dental constituye un problema importante de salud pública en todo el mundo. En México, se destina una gran cantidad de recursos a la rehabilitación dental, que podrían prevenirse mediante hábitos de higiene oral apropiados. Dentro de los indicadores epidemiológicos de riesgo se consideran los antecedentes de índices cariogénicos, que suele ser el predictor más poderoso de caries en jóvenes y adultos (Secretaría de Salud y Asistencia, 2011), siendo la niñez uno de los grupos humanos más vulnerables. Así, el deterioro dental severo es un aspecto importante de atención en esta etapa, el cual se considera una de las enfermedades crónicas más prevalentes en la infancia temprana (Hooley, Skouteris, Biganin, Satur \& Kilpatrick, 2012), especialmente en condición socioeconómica menor (MedinaSolís, Maupomé, Pelcastre-Villafuerte, ÁvilaBurgos, Vallejos-Sánchez \& Casanova-Rosado, 2006) y que afecta el crecimiento de los niños y las niñas, el peso corporal, la calidad de vida e incluso el desarrollo cognitivo y social (Arrow, Raheb \& Miller, 2013).

En la infancia, la dentición temporal permanece en la boca de niños y niñas aproximadamente de los 6 a los 12 años. No obstante, la mayoría de las personas no le dan la importancia necesaria, ya que desconocen que son órganos guía para la erupción de los permanentes, además de la relevancia de mantenerlos sanos y limpios para que la dentición permanente llegue a un ambiente libre de contaminación y enfermedad; por lo que es necesario el seguimiento a los infantes, incluso desde el embarazo. En este sentido, como ha mostrado la investigación (Ramfjord et al., 1982), aunque se cuente con la ayuda profesional, si los pacientes no llevan a cabo un adecuado cuidado de sus dientes de manera sistemática, las medidas preventivas no tendrán resultados positivos a largo plazo.

El entorno que rodea a los niños y niñas debe por tanto potenciar que éstos adquieran los conocimientos y habilidades necesarios que les permitan tener una buena salud en general y de cuidado dental en particular. Los padres de familia son los principales responsables de la salud bucodental en el hogar, ya que ellos determinan si los niños y las niñas llevan a cabo una higiene bucal de manera sistemática (con un enfoque preventivo) o si acuden a la clínica odontológica cuando el problema dental está presente (con un enfoque curativo). Sin 
embargo, no todos cuentan con la preparación para llevarla a cabo de manera adecuada.

Diversas investigaciones muestran que los problemas dentales infantiles están vinculados con las dificultades de acceso a los servicios odontológicos, así como las prácticas y conocimientos deficientes en salud bucal de los padres de familia, que se asocian a su vez con una escasa motivación hacia la salud bucal y con una falta de empoderamiento (Cuartas, Alvar; Maya, Cárdenas, Arias \& Jaramillo, 2002; González-Chávez \& GarcíaRupaya, 2013; González-Penagos, CanoGómez \& Meneses-Gómez, 2015; Hooley et al., 2012), constituyendo un factor de riesgo de orden personal, familiar, social y educativo (Benavente et al., 2012).

De ahí que en los últimos años se ha incrementado el interés por comprender como diferentes determinantes conductuales y psicológicos de los padres se relacionan con la salud bucal de los niños y las niñas. Dentro de éstos se encuentran las creencias sobre la propia capacidad y los hábitos de higiene bucal (Finlayson, Siefert, Ismail \& Sohn, 2007). A pesar de estos esfuerzos, aún son escasos los estudios realizados en este sentido, y no se encontraron trabajos que vinculen estas variables con otras de carácter psicológico, como la intención y control del comportamiento, que permita a los padres y madres de familia afrontar los problemas de la vida y que constituyen un factor importante para mantener un buen estado de salud bucal. Así como tampoco se han llevado a cabo estudios que vinculen estas variables en los padres con las de sus hijos en edad escolar.

El conocimiento de los factores determinantes para la adquisición de estos hábitos en los niños y las niñas y sus padres se considera necesario, especialmente en el contexto mexicano, ya que servirá para implementar y consolidar programas de prevención de salud bucal, mediante una acción participativa que pueda generar un impacto positivo en la disminución de los factores de riesgo y promoción de estilos de vida saludables desde la familia. Teniendo en cuenta que la mayoría de los programas de salud bucal se desarrollan con contenidos muy generales, sin considerar la información recolectada previamente (González-Martínez, Sierra-Barrios \& Morales-Salinas, 2011), ni las variables psicológicas involucradas. Además de que no se realiza un verdadero seguimiento de cada caso en particular para poder valorar el impacto de dichos programas de salud, es decir, no solo se trata de promocionar la salud, es necesario el acompañamiento real de los participantes.

A partir de estos planteamientos, el objetivo principal de este estudio consistió en indagar qué tan capaz se siente el padre o la madre para apoyar a sus hijos en la adquisición de hábitos de higiene bucal como el cepillado dental, el uso de flúor y las revisiones médicas periódicas, así como la intención y control de estos comportamientos y los hábitos de la propia higiene oral. Al mismo tiempo se buscó analizar estos mismos comportamientos y hábitos en sus hijos en edad escolar que asisten a consulta médica.

\section{Revisión de la literatura}

Cepillarse los dientes y remover la placa de manera regular mantiene una boca limpia y saludable. Es por ello que una adecuada higiene bucal es necesaria no sólo desde la primera infancia (6-8 meses de edad), sino incluso desde el embarazo. A partir de los 6 años el cepillado debe realizarse por lo menos dos veces al día, todos los días (Casals-Peidró, 2005), por lo que es necesario dotar a los niños y a las niñas con los medios necesarios para aprender hábitos de higiene bucal y tener un buen control sobre ésta. Uno de los aspectos a fortalecer en este sentido es el auto-cuidado considerado como las decisiones y conductas de la persona encaminadas a mantener un buen estado de salud y a prevenir o disminuir los efectos negativos de esta enfermedad (SotoUgalde, Sexto-Delgado \& Gontán-Quintana, 2014).

Tomando como base un enfoque sociocognitivo, que se ha usado ampliamente para predecir comportamientos relacionados con la salud (Clarkson, Young, Ramsay, Bonner \& Bonetti, 2009), consideramos necesario analizar diferentes variables que inciden en la 
promoción de la conducta de auto-cuidado de los pacientes odontopediátricos, así como el de sus padres, de cara a una buena salud oral.

Desde este marco, una variable clave que incide en el comportamiento es la autoeficacia (Bandura, 1977), considerada como las propias creencias sobre la posesión de las habilidades necesarias y estrategias para obtener los resultados deseados. Así, implícitamente, la autoeficacia hace alusión a las creencias personales específicas sobre la capacidad para llevar a cabo ciertas acciones u obtener los resultados esperados en un contexto o, de otra forma, de ejercer control sobre uno mismo. Bandura (1993) establece que, "las creencias de autoeficacia contribuyen en la motivación de diferentes maneras: determinan las metas que se establece el individuo, la cantidad de esfuerzo que dedica, cuánto tiempo persevera de cara a las dificultades y su recuperación ante los fracasos" (p. 131).

La autoeficacia influye en la elección de actividades y en fijar metas más altas, así como en la persistencia y el esfuerzo dedicado. Especialmente, cuando se enfrentan a obstáculos, las personas con un alto sentido de autoeficacia trabajan más duro y persisten más tiempo que aquellos que dudan de sus capacidades (Bandura \& Cervona, 1983). El grado de percepción de autoeficacia está definido a su vez por un dominio específico y depende del contexto particular (Zimmerman, 2000). En el contexto de la salud bucal, la autoeficacia de los padres implica la creencia de su propia capacidad en cuidar de la salud oral de sus hijos (Östberg, Skeie, Skaare \& Espelid, 2016).Se ha evidenciado que la autoeficacia parental es un predictor importante de la salud bucal de los niños y las niñas (Finlayson et al., 2007), existiendo un impacto positivo en la disminución de la caries infantil cuando los padres o adultos cercanos se involucran para que sus hijos/hijas sean conscientes de la importancia de mantener buenos hábitos de cepillado dental (Farooqi, Khabeer, Moheet, Khan, Farooq \& ArRejaie, 2015). La asistencia, supervisión y ejemplo de los adultos cercanos al niño, tiene por tanto un papel primordial en el cuidado adecuado de los dientes de manera sistemática, de cara a la prevención de caries dental en los niños y las niñas; ya que, como indican estudios previos, educar a los padres en métodos de prevención contra la caries, reduce su prevalencia (Arrow et al., 2013; Osborn, Paasche-Orlow, Bailey \& Wolf, 2011).

En este mismo sentido, uno de los modelos psicológicos más difundidos para predecir el cambio de comportamiento es la teoría del control de la acción (Gollwitzer, 1999; Kuhl, 1985), la cual propone que la motivación por sí sola es insuficiente para mantener la intención, siendo necesario además tener el compromiso y la determinación para llevarla a cabo. Especialmente las intenciones difíciles requieren del autocontrol (control de la acción), ante otras alternativas, sobre todo cuando el logro requiere el involucramiento en comportamientos a más largo plazo o no muy placenteros, lo que genera un conflicto de acercamiento-evitación. Las intenciones difíciles deben ser protegidas por tanto contra otras alternativas, pensamientos debilitantes $\mathrm{o}$ surgimiento de emociones no deseadas.

Desde este enfoque, se considera necesario en primer lugar establecer una intención y una meta a lograr; en segundo lugar se considera que existen dificultades, deseos o alternativas al logro de esa meta; por último se evalúa el esfuerzo sostenido para llevar a cabo el objetivo planteado (autocontrol). El hacer un plan de manera explícita sobre dónde y cuándo se desarrollará el comportamiento, incrementa la posibilidad de su implementación (Clarkson et al., 2009). Asimismo, el control de la acción percibido estará en función de las creencias sobre los factores que facilitan o restringen el comportamiento como por ejemplo las habilidades y capacidades personales (Bonetti, Johnston, Clarkson \& Turner, 2013).

En el cuidado dental, en particular, las personas deben realizar esfuerzos para organizar sus acciones de manera sistemática, que les permita construir y mantener en el tiempo buenos hábitos de higiene bucal, a pesar de las dificultades. Es por ello que, el conocimiento de los factores anteriores puede contribuir a la generación de programas que promuevan de manera explícita las habilidades para el autocuidado, con un enfoque de agencia y autocontrol desde la infancia temprana. 
En cuanto a los hábitos respecta, éstos pueden definirse como "la práctica o costumbre que se adquiere por la repetición continua de un mismo acto, inicialmente es un acto voluntario o consciente, que se convierte en involuntario o inconsciente cuando se arraiga" (León, Carvajal, Pérez \& Rodríguez, 2014, p.1). Entre los buenos hábitos de higiene oral se encuentran el cepillado correcto de los dientes diariamente, por lo menos de dos a tres veces al día; aunque idealmente después de cada ingesta, enfatizando el cepillado por la noche, el uso de hilo dental para remover la placa alojada en los espacios interdentales, el uso de enjuague bucal ocasional y realizar visitas al odontólogo dos veces al año (Casals-Peidró, 2005; SoriaHernández, Molina \& Rodríguez, 2008).

Varios investigadores (Martins, Oliveira, Pordeus \& Paiva, 2011) han encontrado que los hábitos de la salud oral de los niños y las niñas no están influenciados por el proceso de aprendizaje en la escuela. Sin embargo, un estudio realizado en escuelas de Arabia Saudita (Farooqi et al., 2015) reveló el impacto positivo de los hábitos de cepillado sobre la caries cuando se encuentran involucrados los niños y las niñas, los maestros y los padres en la concientización de la importancia de los hábitos del cepillado. Otros estudios (Finlayson et al., 2007; Freire, Luiz, De Souza \& PuppinRontanid, 2012; Okada et al., 2002) sostienen que los comportamientos de salud de los niños y las niñas y sus resultados están influenciados en gran medida por el conocimiento, las creencias y los hábitos de sus padres, los cuales afectan su higiene oral.

De hecho, los niños y las niñas todavía están desarrollando sus hábitos de higiene bucal y generalmente no son capaces de cepillarse, enjuagarse y escupir correctamente (Bentley, Ellwood \& Davies, 1999), por lo que se han encontrado mejores hábitos de cepillado de dientes en las madres que en sus hijos pequeños (Martins et al., 2011). Asimismo, una revisión sistemática de diversas investigaciones realizadas entre 1980 y 2012 (Freire et al., 2012), mostró que factores como el nivel socioeconómico, el grado de estudios, la situación laboral o la edad de los padres de familia son factores clave que tienen un impacto particular en los comportamientos y resultados de salud oral en sus hijos en edad infantil.

Por lo anteriormente expuesto se plantean las siguientes hipótesis:

H1. El/la paciente odontopediátrico/a percibe una menor autoeficacia para la higiene dental que su progenitor/a.

$\mathrm{H} 2$. El/la paciente odontopediátrico/a tiene hábitos de higiene bucal menos desarrollados que su progenitor/a.

H3. El/la paciente odontopediátrico/a cuenta con una menor intención de involucrarse en actividades de higiene bucal que su progenitor/a.

H4. El/la paciente odontopediátrico/a tiene menor control de su higiene bucal que su progenitor/a.

\section{Metodología}

Se desarrolló una investigación exploratoria de tipo cuantitativo, descriptivo y transversal. La población estuvo constituida por padres y madres de familia, así como sus hijos que asistieron a la clínica odontológica universitaria en una ciudad al centro de México, durante el período de abril a mayo de 2014. Se trató de un estudio censal, ya que la aplicación de los instrumentos se realizó al total de pacientes odontopediátricos que asistieron a consulta durante el periodo de estudio.

Criterios de inclusión:

- Niños y niñas con edad entre 6 y 13 años que contaran con cita odontológica

- Padre o madre acompañante del infante a la clínica universitaria

- Consentimiento informado por parte de los padres de familia de su participación y la de sus hijos

Sujetos de estudio. El estudio involucró a 43 pacientes odontopediátricos, entre 6 y 13 años de edad, y 36 padres de familia, entre 21 y 47 años de edad, que acompañaban a sus hijos a recibir tratamiento.

Instrumentos. Se desarrollaron dos instrumentos para ser aplicados de manera individual a los niños/niñas y a su padre/madre, respectivamente, los cuales fueron adaptados de instrumentos previamente validados $y$ 
publicados en las diferentes variables de estudio, los cuales se describen a continuación:

Autoeficacia para la higiene dental. The Perceived Health Competence Scale (Dempster $\&$ Donnelly, 2008; $\alpha=.91)$ se adaptó para medir la agencia personal para el cuidado oral de los/ las niños/niñas y la de sus padres/madres (e. g., "No importa cuánto lo intente, mis dientes no quedan lo suficientemente limpios", "Sé qué debo hacer para mantener mis dientes limpios").

Hábitos de higiene bucal. La versión en español (Gutiérrez-Sánchez \& Pino-Juste, 2011; $\alpha=.96)$ del Self-Report Index of Habit Strength (SRHI; Verplanken \& Orbell, 2003) se adaptó para evaluar los hábitos diarios de los/ las niños/niñas y la de sus padres/madres para mantener una buena higiene bucal (e. g., "Me cepillo los dientes después de cada comida", "Me siento mal si no me cepillo los dientes después de comer").

Intención del comportamiento. Las intenciones de los/las niños/niñas y la de sus padres/madres para llevar a cabo actividades que promueven la higiene bucal se evaluaron a partir de la escala propuesta por Caudroit, Boiché y Stephan (2014; $\alpha=.93)$, que mide las intenciones para involucrarse en actividades físicas (e. g., "Quiero aprender a cepillar correctamente mis dientes", "Quiero aprender a cuidar mis dientes" para el caso de los niños/ niñas y "quiero enseñar a mi hijo/a a cepillarse correctamente los dientes", "Estoy interesado/a en buscar información sobre salud bucal" para el caso de los padres/madres).

Control de la acción. Se usó el Habitual self-control questionnaire (HSCQ de Schroder, Ollis \& Davies, 2013; $\alpha=.81$ ) para medir la habilidad percibida por los niños/niñas y por sus padres/madres para mantener las intenciones a pesar de las dificultades o las distracciones (e. g., "Termino una tarea aunque no me guste", "Aunque quiera terminar una tarea, me distraigo").

El cuestionario para padres/madres consistió de 38 ítems y el de los niños/niñas de 30 ítems. Los reactivos de ambos cuestionarios se evalúan mediante una escala tipo Likert que va de 1(totalmente en desacuerdo) a 5(totalmente de acuerdo). Las variables evaluadas en los instrumentos se indican a continuación (ver Tabla 1).

Tabla 1. Variables evaluadas en pacientes odontopediátricos y padres de familia.

\begin{tabular}{ll}
\hline \multicolumn{1}{c}{ Pacientes infantiles } & \multicolumn{1}{c}{ Padres de familia } \\
\hline Autoeficacia & Autoeficacia \\
Hábitos de higiene bucal & Hábitos de higiene bucal \\
Intención del comportamiento & Intención del comportamiento \\
Control de la acción & Control de la acción \\
\hline
\end{tabular}

Fuente: Elaboración propia.

Procedimiento. Los instrumentos fueron aplicados de manera individual a cada niño/ niña y a su padre/madre que lo acompañaba a recibir tratamiento, en las instalaciones de la clínica universitaria, durante el horario de consulta. Teniendo en cuenta que los niños y las niñas pequeños (6 años de edad) no tienen un dominio de las habilidades de lecto-escritura, una de las investigadoras leyó en voz alta las preguntas del cuestionario a los niños y las niñas, anotando sus respuestas en el instrumento. Al resto de los niños y niñas sólo se les leyeron las instrucciones, así como al padre o madre acompañante.

Para el análisis de los datos (de padres de familia y de sus respectivos hijos/hijas), se utilizó la prueba de los rangos con signo de Wilcoxon, para las variables autoeficacia, hábitos, intención de comportamiento y control de la acción. Los análisis comparativos por grupo (por edad, nivel de estudios de los padres/ madres, trabajo de los padres/madres fuera del 
hogar) se realizaron mediante tau c de Kendall. Los análisis estadísticos se desarrollaron utilizando el software SPSS versión 20.

\section{Análisis de resultados}

Análisis descriptivo. Del total de pacientes odontopediátricos participantes en la investigación, $56.1 \%$ son niñas y $43.9 \%$ niños, con una edad promedio de 8.9 años. Respecto al padre o madre encuestado, $75.6 \%$ son mujeres y $24.4 \%$ son hombres, con un promedio de 34.64 años de edad. La mayor parte tiene 2 hijos (43.9\%) y en la mayoría de los casos ambos padres trabajan fuera del hogar (45.7\%), seguido por los hogares donde solamente el padre trabaja fuera del hogar $(40 \%)$.

En cuanto al nivel educativo de los progenitores, el $71.4 \%$ de las madres cuenta con educación básica, media o técnica-superior, $22.9 \%$ tiene estudios universitarios y sólo 5.7\% no tiene estudios. El caso de los padres es muy semejante; $72.7 \%$ cuenta con enseñanza básica, media o estudios técnicos, $21.5 \%$ tiene estudios universitarios, y sólo $6.1 \%$ no tiene estudios. En la tabla 2 se sintetiza la información relacionada con el perfil de los participantes del estudio.

Tabla 2. Perfil de pacientes odontopediátricos y padres de familia.

\begin{tabular}{|c|c|c|c|c|c|}
\hline \multicolumn{6}{|c|}{ Pacientes odontopediátricos } \\
\hline Edad & Frecuencia & $\%$ & Sexo & Frecuencia & $\%$ \\
\hline 6 & 6 & 14.6 & Niño & 18 & 43.9 \\
\hline 7 & 4 & 9.8 & Niña & 23 & 56.1 \\
\hline 8 & 12 & 29.3 & $(\mathrm{n}=41)$ & & \\
\hline 9 & 2 & 4.9 & & & \\
\hline 10 & 6 & 14.6 & & & \\
\hline 11 & 6 & 14.6 & & & \\
\hline 12 & 3 & 7.3 & & & \\
\hline 13 & 2 & 4.9 & & & \\
\hline$(n=41)$ & & & & & \\
\hline \multicolumn{6}{|c|}{ Padres } \\
\hline Edad & Frecuencia & $\%$ & Sexo & Frecuencia & $\%$ \\
\hline $21-25$ & 2 & 4.9 & Masculino & 10 & 24.4 \\
\hline $26-30$ & 10 & 24.4 & Femenino & 31 & 75.6 \\
\hline $31-35$ & 14 & 34.1 & $(\mathrm{n}=41)$ & & \\
\hline $36-40$ & 5 & 12.2 & & & \\
\hline $41-45$ & 9 & 2.2 & & & \\
\hline $46-50$ & 1 & 2.4 & & & \\
\hline$(n=41)$ & & & & & \\
\hline
\end{tabular}

\begin{tabular}{cccccc|} 
No. Hijos & Frecuencia & $\mathbf{\%}$ & $\begin{array}{c}\text { Actividades } \\
\text { laborales fuera } \\
\text { del hogar }\end{array}$ & Frecuencia & $\%$ \\
1 & 5 & 12.2 & Sólo el padre & 14 & 40 \\
2 & 18 & 43.9 & Sólo la madre & 5 & 14.3 \\
3 & 9 & 2.2 & Ambos padres & 16 & 45.7 \\
4 & 5 & 12.2 & $(\mathrm{n}=35)$ & & \\
5 & 4 & 9.8 & & & \\
$(\mathrm{n}=41)$ & & & & &
\end{tabular}




\begin{tabular}{|c|c|c|c|c|c|}
\hline $\begin{array}{c}\text { Nivel de } \\
\text { estudios de la } \\
\text { madre }\end{array}$ & Frecuencia & $\%$ & $\begin{array}{c}\text { Nivel de } \\
\text { estudios del } \\
\text { padre }\end{array}$ & Frecuencia & $\%$ \\
\hline Sin estudios & 2 & 5.7 & Sin estudios & 2 & 6.06 \\
\hline $\begin{array}{c}\text { Enseñanza } \\
\text { básica }\end{array}$ & 9 & 25.7 & $\begin{array}{l}\text { Enseñanza } \\
\text { básica }\end{array}$ & 8 & 24.2 \\
\hline $\begin{array}{l}\text { Enseñanza } \\
\text { media }\end{array}$ & 11 & 31.4 & $\begin{array}{l}\text { Enseñanza } \\
\text { media }\end{array}$ & 11 & 33.3 \\
\hline $\begin{array}{l}\text { Estudios } \\
\text { técnicos }\end{array}$ & 5 & 14.3 & $\begin{array}{l}\text { Estudios } \\
\text { técnicos }\end{array}$ & 5 & 15.2 \\
\hline $\begin{array}{c}\text { Estudios } \\
\text { universitarios } \\
(\mathrm{n}=35)\end{array}$ & 8 & 22.9 & $\begin{array}{c}\text { Estudios } \\
\text { universitarios } \\
(\mathrm{n}=33)\end{array}$ & 7 & 21.2 \\
\hline
\end{tabular}

Fuente: Elaboración propia.

Prueba del signo. Dada la naturaleza ordinal de las percepciones obtenidas a partir de dos muestras dependientes, de los pacientes pediátricos de la clínica y de sus padres, se realizó la prueba de los rangos con signo de Wilcoxon para las variables autoeficacia, hábitos, intención de comportamiento y control de la acción. Esta prueba examina el signo de la diferencia entre pares de datos (entre el primer sujeto en la primera muestra y el mismo sujeto en la segunda muestra, hasta el n-ésimo par $\mathrm{y}$ en base a las medianas). Cuando no existe diferencia significativa entre las dos muestras, la cantidad de diferencias positivas y negativas será igual o muy similar entre ambas muestras, es así que cuando la contrastación de la hipótesis conlleva a un resultado no significativo $(p>$
$0.05)$, se concluye que no es posible asumir que las dos muestras difieren (Garson, 2008).

Los resultados evidencian diferencias significativas entre los dos grupos, pacientes pediátricos y sus padres, respecto a tres de las cuatro variables estudiadas: autoeficacia $(\mathrm{p}=0.010)$, hábitos $(\mathrm{p}=0.000)$ e intención de comportamiento $(p=0.000)$. Los pacientes odontopediátricos presentaron mayor autoeficacia que su progenitor encuestado, en cambio, los padres presentaron mejores respuestas en hábitos de higiene bucal e intención de comportamiento (Tabla 3). No obstante, el control de la acción resultó no significativo $(p=0.069)$ entre la comparación de pacientes pediátricos y sus padres.

Tabla 3. Comparación de percepciones entre pacientes y sus padres.

\begin{tabular}{cccc}
\hline Variable & $\begin{array}{c}\text { Mediana } \\
\text { pacientes }\end{array}$ & $\begin{array}{c}\text { Mediana } \\
\text { Padres }\end{array}$ & Significancia \\
\hline Autoeficacia & 2.85 & 2.33 & 0.010 \\
\hline Hábitos de higiene bucal & 2.28 & 2.87 & 0.000 \\
\hline Intención de comportamiento & 2.60 & 3.40 & 0.000 \\
\hline Control de la acción & 3.18 & 3.0 & 0.069 \\
\hline
\end{tabular}

Fuente: Elaboración propia.

\section{Tabulación cruzada}

Edad. El análisis de los cruces considerando seis grupos de edad y las variables de estudio se realizaron mediante tau c de Kendall. Los resultados evidenciaron que no hay asociación entre la edad de los padres y las percepciones de autoeficacia, hábitos, control de la acción e intención de comportamiento de los pacientes odontopediátricos. Asimismo, el análisis de edad y las variables de estudio de los propios padres tampoco evidenció significancia. Por lo 
anterior, puede decirse que la edad de los padres no se asocia con las variables de estudio en los pacientes, ni de los propios padres.

Nivel de estudios de la madre y el padre. El análisis mostró que no hay asociación entre el nivel de estudios de la madre o el padre y las percepciones de autoeficacia, hábitos, control de la acción e intención de comportamiento de los pacientes odontopediátricos. Tampoco se identificaron asociaciones significativas entre nivel de estudios de la madre o el padre y las variables de análisis.

Trabajo fuera del hogar. El análisis mostró que no hay asociación entre el trabajo fuera del hogar y las percepciones de autoeficacia, hábitos, control de la acción e intención de comportamiento tanto de los pacientes odontopediátricos como del progenitor encuestado.

\section{Discusión}

El propósito principal de este estudio consistió en indagar qué tan capaces se sienten los padres de familia para apoyar a sus hijos en la adquisición de hábitos de higiene bucal, así como la intención y control de estos comportamientos y los hábitos de la propia higiene oral. Además de analizar estos mismos comportamientos y hábitos en sus hijos en edad escolar que asisten a consulta médica.

Los resultados evidencian diferencias significativas entre el grupo de pacientes odontopediátricos y el de sus padres, respecto a tres de las cuatro variables estudiadas: autoeficacia, intención de comportamiento y hábitos de higiene bucal. Los pacientes odontopediátricos presentaron mayor autoeficacia que su progenitor encuestado; en cambio, los padres presentaron mejores respuestas en intención de comportamiento y hábitos de higiene bucal. No obstante, el control de la acción resultó no significativo en la comparación de pacientes pediátricos y sus padres. A partir de estos hallazgos se comprueban dos de las cuatro hipótesis formuladas:

H2. El/la paciente odontopediátrico/a tiene hábitos de higiene bucal menos desarrollados que su progenitor/a.
H3. El/la paciente odontopediátrico/a cuenta con una menor intención de involucrarse en actividades de higiene bucal que su progenitor/a.

No obstante no se cumplieron dos de las hipótesis:

H1. El/la paciente odontopediátrico/a percibe una menor autoeficacia para la higiene dental que su progenitor/a.

H4. El/la paciente odontopediátrico/a tiene menor control de su higiene bucal que su progenitor/a.

Es de llamar la atención que los niños y las niñas del estudio perciban una mayor autoeficacia que sus padres, ya que no obstante la percepción de competencia y expectativas de éxito en la primera y segunda infancia (antes de los 6 años) son desproporcionadas y sobrevaloradas, éstas comienzan a ser congruentes con los desempeños personales en la tercera infancia -entre 7 y 12 años de edad(Alonso-Tapia, 2005), lo cual indica que los niños y niñas del estudio aún se encuentran en este proceso. Es importante señalar en este sentido, que la percepción de autoeficacia por parte de los padres y madres de familia se considera un mecanismo determinante para que puedan guiar a los niños y las niñas a que realicen una adecuada higiene bucal de manera autónoma, ya que los progenitores constituyen el principal punto de referencia para el desarrollo infantil (Henao \& García, 2009). La percepción de autoeficacia de padres y madres puede incidir por tanto en la elección de estrategias para el cuidado bucal personal, así como en el esfuerzo dedicado para la supervisión del cepillado de sus hijos (Finlayson et al., 2007; Ostberg et al., 2016) que permita afianzar las buenas prácticas de higiene bucal. De ahí la importancia de que los profesionales de la salud lleven a cabo capacitaciones participativas, a fin de promover los conocimientos, las creencias de autoeficacia y habilidades y prácticas orales adecuadas de los padres y madres sobre la salud bucal para un óptimo desarrollo de sus familias.

Como se esperaba, los padres de familia presentan una mayor intención de comportamiento y hábitos de higiene bucal que sus hijos, lo cual corrobora el que los niños y las niñas aún se encuentran en un proceso de 
desarrollo de hábitos de higiene bucal (Bentley et al., 1999) y requieren de una supervisión y apoyo cercano de sus progenitores. Asimismo, los hallazgos coinciden con otras investigaciones que han obtenido mejores hábitos de cepillado de dientes en las madres que en sus hijos pequeños (Martins et al., 2011).

No obstante, el que no haya diferencias significativas en el control de la acción entre los pacientes odontopediátricos y sus padres, indica la necesidad de realizar trabajo de intervención con ambos grupos, a fin de potenciar el que tengan la determinación para mantener un esfuerzo sostenido ante las dificultades que los puedan desviar del logro de su meta en el largo plazo, en este caso de una buena salud bucal. Educar a los padres en métodos de prevención contra la caries, a partir de una preparación psicológica, que involucre conocimientos, intenciones y control del comportamiento, puede contribuir de manera significativa a su involucramiento para el desarrollo de hábitos y prácticas de higiene oral personal y de sus hijos, que puedan eventualmente incidir en la reducción de la prevalencia de afecciones dentales (Arrow et al., 2013). Consideramos que una aproximación educativa que involucre a toda la familia en la promoción de estrategias autorregulatorias -hacer un plan de manera explícita, supervisar su implementación y evaluar los resultados- desde una perspectiva de control de la acción (Gaeta \& Herrero, 2009) puede incrementar la posibilidad de este logro.

Los padres de familia, como principales responsables de la salud bucodental en el hogar, son un factor de influencia determinante en la adquisición y desarrollo de hábitos de higiene oral en sus hijos (Finlayson et al., 2007), por lo que el compromiso y la determinación de los progenitores para llevar a cabo una higiene oral adecuada y el esfuerzo sostenido para mantener hábitos saludables, puede contribuir a que sean una guía y ejemplo para el involucramiento de sus hijos en la adquisición y desarrollo de hábitos de higiene oral a largo plazo.

Por otro lado, los resultados evidenciaron que no hay asociación entre la edad de los padres y las percepciones de autoeficacia, hábitos, control de la acción e intención de comportamiento de sus hijos. Asimismo, el análisis de edad y las variables de estudio de los propios padres tampoco evidenció significancia. Por lo anterior, puede decirse que la edad de los padres no se asocia con las variables de estudio en los pacientes ni de los propios padres, lo cual coincide con otros estudios que no encontraron una relación significativa entre la edad de los padres y sus conocimientos y actitudes hacia la higiene dental de sus hijos (Williams, Whittle \& Gatrell, 2002).

Respecto al trabajo de los padres fuera del hogar el análisis mostró que no hay asociación entre éste y las percepciones de autoeficacia, hábitos, control de la acción e intención de comportamiento, tanto de los pacientes odontopediátricos como del progenitor encuestado. En un estudio similar (Nieto-García, Nieto-García, Lacalle-Remigio \& Abdel-Kader, 2001) con escolares españoles de 7 a 14 años de edad, se encontró una mayor salud oral en los niños y las niñas con padres empleados que aquellos con padres desempleados. En el caso del presente estudio, el que todos los padres de familia perciban un ingreso, independientemente del tipo de trabajo desempeñado, no representa un factor diferenciador. Consideramos que estos factores de estudio están relacionados más bien con la falta de educación en prevención de la población. Estos datos nos llevan a una indagación más profunda en este sentido en futuras investigaciones.

De igual forma, no se encontró una relación significativa entre el nivel de estudios de los padres y las percepciones de autoeficacia, hábitos, control de la acción e intención de comportamiento de los pacientes odontopediátricos. Tampoco se identificaron asociaciones significativas entre nivel de estudios de la madre o el padre y las variables de análisis. En este sentido, Williams et al. (2002) encontraron una relación significativa entre el nivel educativo de los padres vinculado con un nivel socioeconómico bajo y sus conocimientos y actitudes hacia la higiene dental. Asimismo, Navas, Rojas, Zambrano, Álvarez, Santana y Viera (2002), hallaron una relación significativa entre la educación de los padres y el nivel de caries en sus hijos en edad pre-escolar. Por su parte, otro estudio con 2,939 
niños y niñas mexicanos entre 6 y 12 años de edad (Medina-Solís et al., 2006) mostró que el nivel socioeconómico de la familia es un factor determinante en la incidencia de caries infantil. Los datos anteriores nos muestran la complejidad de los factores socio-culturales en los comportamientos y hábitos de higiene bucal, sobre todo al tratarse de grupos en desventaja social (González-Penagos et al., 2015; Williams et al., 2002), lo que nos conduce a una mayor profundización en el estudio de estas variables vinculadas al comportamiento y hábitos de los padres de familia y de sus hijos en edad infantil.

Existen limitantes en el presente estudio que es necesario mencionar. En primer lugar, entendemos que al serun estudio exploratorio con una muestra pequeña, no podemos generalizar los hallazgos. Asimismo, los datos provienen de la aplicación de encuestas a los niños y niñas que asisten a consulta médica en la clínica universitaria y su padre/madre acompañante, por lo que no se obtuvo información sobre otros miembros de la familia que pudieran incidir en el desarrollo de habilidades y comportamientos de los menores respecto a su cuidado dental.

\section{Conclusiones}

La salud bucal constituye un factor importante para la salud personal en general, con consecuencias fisiológicas, psicológicas y sociales importantes (Arrow et al., 2013). Es por ello necesario orientar en hábitos de higiene oral saludables desde el entorno familiar, así como sobre los factores que previenen las enfermedades bucales. Sin embargo, estos esfuerzos no tendrán resultados a largo plazo si las personas no se sienten capaces para llevarlos a cabo, no tienen la intención y el control para su realización o no saben cómo generar hábitos de higiene bucal saludables.

Los datos encontrados en el presente estudio se consideran importantes, ya que para que una intervención pueda tener resultados favorables es necesario conocer las necesidades en salud de la población a la que se atiende, así como a los adultos responsables de la adquisición y promoción de estas habilidades y hábitos. De esta forma, el apoyo, supervisión y ejemplo de los adultos cercanos al niño/niña tienen un papel fundamental para que estos últimos se responsabilicen de su autocuidado dental y desarrollen las estrategias necesarias para la autogestión de sus propios procesos de manera gradual y sistemática. A partir de nuestros hallazgos se enfatiza la necesidad de centrar la atención en acciones de prevención y promoción de hábitos higiénicos bucales en la infancia como parte de una rutina diaria. En este marco, los odontólogos desempeñan un rol relevante en la prevención primaria de los problemas dentales de los niños y las niñas a través de tratamientos preventivos, evaluación de riesgos y orientación para los padres sobre el desarrollo oral, la prevención de caries y la salud oral en general (Huebner \& Riedy, 2010).

Dado el modelo actual de familia, en donde muchas veces ambos padres trabajan fuera del hogar, hay que brindar educación en prevención y en la adquisición de habilidades autorregulatorias a los padres y a aquellas personas que hacen el rol de cuidadores de los menores, por ejemplo: abuelos, tíos, etc., a fin de que estén informados, se motiven y adquieran las competencias necesarias para el cuidado de su propia salud bucal y la de los infantes de manera sistemática. De esta forma, la concepción de los niños y las niñas como sujetos activos en su propio desarrollo, toma relevancia a partir de la consideración de los padres/madres como ejes y no como intermediarios en la atención de sus hijos/ hijas, valorando los conocimientos y el sentido común de los adultos significativos por parte de los funcionarios de salud (Peñaranda, 2002). Consideramos que el presente estudio puede servir de guía a los profesionales, especialmente en la clínica universitaria de estudio, para generar programas preventivos que fortalezcan la salud oral de niños/niñas y adultos, mediante una práctica participativa.

\section{Lista de referencias}

Alonso-Tapia, J. (2005). Motivar en la escuela, motivar en la familia. Madrid: Morata.

Arrow, P.; Raheb, J. \& Miller, M. (2013). Brief oral health promotion intervention among parents of young children to reduce early childhood dental decay. $B M C$ 
Public Health, 13 (1), pp. 245-253. Doi: 10.1186/1471-2458-13-245.

Bandura, A. (1977). Self-efficacy. Toward a Unifying Theory of Behavioral Change. Psychological Review, 84 (2), pp. 191-215. Bandura, A. (1993). Perceived self-efficacy in cognitive development and functioning. Educational Psychologist, 28, pp. 117-148. Doi: 10.1207/s15326985ep2802_3

Bandura, A. \& Cervone, D. (1983). SelfEvaluative and Self-Efficacy Mechanisms Governing the Motivational Effects of Goal Systems. Journal of Personality and Social Psychology, 45 (5), pp. 1017-1028. Doi: 10.1037/0022-3514.45.5.1017.

Benavente, L.; Chein, S.; Campodónico, C.; Palacios, E.; Ventocilla, M.; Castro, A.; Huapaya, O.; Álvarez, M.; Paulino, W. \& Espetia, A. (2012). Nivel de conocimientos en salud bucal de las madres y su relación con el estado de salud bucal del niño menor de cinco años de edad. Odontología Sanmarquina, 15 (1), pp. 14-18.

Bentley, E. M.; Ellwood, R. P. \& Davies, R. M. (1999). Oral Hygiene: Fluoride ingestion from toothpaste by Young children. British Dental Journal, 186 (9), pp. 460-462. Doi: 10.1038/sj.bdj.4800140.

Bonetti, D.; Johnston, M.; Clarkson, J. \& Turner, S. (2013). Applying multiple models to predict clinicians' behavioural intention and objective behaviour when managing children's teeth. Psychology \& Healt, 24 (7), pp. 843-860. Doi: 10.1080/08870440802108918.

Casals-Peidró, E. (2005). Hábitos de higiene oral en la población escolar y adulta española. Rcoe, 10 (4), pp. 389-401. Doi: 10.4321/S1138-123X2005000400002.

Caudroit, J.; Boiché, J. \& Stephan, Y. (2014). The role of action and coping planning in the relationship between intention and physical activity: A moderated mediation analysis. Psychology and Health, 29 (7), pp. 768-780. Doi:10.1080/08870446.2014 .884223 .

Clarkson, J. E.; Young, L.; Ramsay, C. R.; Bonner, B. C. \& Bonetti, D. (2009). How to Influence Patient Oral Hygiene Behavior Effectively. Journal of Dental
Research, 88 (10), pp. 933-937. Doi: 10.1177/0022034509345627.

Cuartas, J.; Alvar, A.; Maya, A.; Cárdenas, J.; Arias, M. \& Jaramillo, A. (2002). Relación entre percepción de los padres sobre el tratamiento odontológico y sus hábitos de higiene oral, con la historia de caries dental de sus hijos, entre 3 y 5 años de edad. Revista CES Odontología, 15 (1), pp. 1318.

Dempster, M. \& Donnelly, M. (2008). Validity of the Perceived Health Competence Scale in a UK primary care setting. Psychology, Health \& Medicine, 13 (1), pp. 123-127. Doi: 10.1080/13548500701351984.

Farooqi, F. A.; Khabeer, A.; Moheet, I. A.; Khan, S. Q.; Farooq, I. \& ArRejaie, A. S. (2015). Prevalence of dental caries in primary and permanent teeth and its relation with tooth brushing habits among schoolchildren in Eastern Saudi Arabia. Saudi Medical Journal, 36 (6), pp. 737-742.

Finlayson, T.; Siefert, K.; Ismail, A. \& Sohn, W. (2007). Psychosocial factors and early childhood caries among low-income African-American children in Detroit. Community Dental Oral Epidemiology, 35, pp. 439-448. Doi: 10.1111/j.16000528.2006.00352.x.

Freire, A. R.; Luiz, F.; De Souza, T. \& PuppinRontanid, R. M. (2012). Influence of family environment on childrens' oral health: a systematic review. Jornal de Pediatria, 89 (2), pp. 116-123.

Gaeta, M. L. \& Herrero, M. L. (2009). Influencia de las estrategias volitivas en la autorregulación del aprendizaje. Estudios de Psicología, 30 (1), pp. 73-88. Doi: 10.1174/021093909787536317.

Garson, D. (2008). Pruebas de importancia para dos muestras dependientes: Mcnemar, homogeneidad marginal, signo y pruebas de Wilcoxon. Recuperado de: http://faculty. chass.ncse.edu/garson/PA765/mcnemar. htmsigntest

Gollwitzer, P. (1999). Implementation intentions: Strong effects of simple plans. American Psychologist, 54 (7), pp. 493503. Doi: 10.1037/0003-066X.54.7.493. 
González-Chávez, R. \& García-Rupaya, C. (2013). Comparación de dos programas educativos y su influencia en la salud bucal de preescolares. Kiru, 10 (1), pp. 18-25.

González-Martínez, F.; Sierra-Barrios, C. \& Morales-Salinas, L. (2011). Conocimientos, actitudes y prácticas en salud bucal de padres y cuidadores en hogares infantiles, Colombia. Salud Pública de México, 53 (3), pp. 247-257.

González-Penagos, C.; Cano-Gómez, M. \& Meneses-Gómez, E. J.(2015). Percepciones en salud bucal de los niños y niñas. Revista Latinoamericana de Ciencias Sociales, Niñez y Juventud, 13 (2), pp. 715-724. Doi: 10.11600/1692715x.13211270314.

Gutiérrez-Sánchez, A. \& Pino-Juste, M. (2011). Validación de la versión en español de las propiedades psicométricas de la Escala Self-Report Habit Index (SRHI) para medir hábitos de ejercicio físico. Revista Española de SaludPública, 85, pp. 363-371. Doi:10.1590/S1135-57272011000400005.

Henao, G. \& García, M. C. (2009). Interacción familiar y desarrollo emocional en niños y niñas. Revista Latinoamericana de Ciencias Sociales, Niñez y Juventud, 7 (2), pp. 785-802.

Hooley, M.; Skouteris, H.; Biganin, C.; Satur, J. \& Kilpatrick, N. (2012). Parental influence and the development of dental caries in children aged 0-6 years: A systematic review of the literature. Journal of dentistry, 40 (11), pp. 873-885. Doi: 10.1016/j.jdent.2012.07.013.

Huebner, C. E. \& Riedy, C. A. (2010). Behavioral determinants of brushing young children's teeth: implications for anticipatory guidance. Pediatric dentistry, 32 (1), pp. 48-55.

Kuhl, J. (1985). Volitional mediator of cognition-behavior consistency: selfregulatory processes and action versus state orientation. En J. Kuhl \& J. Beckmann (eds.) Action control: From cognition to behavior, (pp. 101-128). Berlin: Springer.

León, J.; Carvajal, Y.; Pérez, N. \& Rodríguez, O. (2014). Hábitos bucales deformantes y su posible influencia sobre el plano poslácteo en niños con dentición temporal.
Mediciego, 20 (1), pp. 3-9. Recuperado de: http://bvs.sld.cu/revistas/mciego/vol20 Supl\%201 14/pdf/T8.pdf

Martins, C. C.; Oliveira, M. J.; Pordeus, I. A. \& Paiva, S. M. (2011). Comparison between observed children's tooth brushing habits and those reported by mothers. BMC oral health, 11 (1), pp. 22-33.

Medina-Solís, C.; Maupomé, G.; PelcastreVillafuerte, B.; Ávila-Burgos, L.; VallejosSánchez, A. \& Casanova-Rosado, A. (2006). Desigualdades socioeconómicas en salud bucal: caries dental en niños de seis a 12 años de edad. Revista de Investigación Clínica, 58 (4), pp. 296-304.

Navas, R.; Rojas, T.; Zambrano, O.; Álvarez, C.; Santana, Y. \& Viera, N. (2002). Salud bucal en preescolares: su relación con las actitudes y nivel educativo de los padres. Interciencia, 27 (11), pp. 631-634.

Nieto-García, V. M.; Nieto-García, M. A.; Lacalle-Remigio, J. R. \& Abdel-Kader, M. L. (2001). Salud oral de los escolares de Ceuta: influencias de la edad, el género, la etnia y el nivel socioeconómico. Revista española de salud pública, 75 (6), pp. 541 550.

Okada, M.; Kawamura, M.; Kaihara, Y.: Matsuzaki, Y.; Kuwahara, S.; Ishidori, H. \& Miura, K. (2002). Influence of parents' oral health behavior on oral health status of their school children: an exploratory study employing a causal modelling technique. International Journal of Pediatric Dentistry, 12 (2), pp. 101-108. Doi: 10.1046/j.1365-263X.2002.00338.x.

Osborn, C. Y.; Paasche-Orlow, M. K.; Bailey S. C. \& Wolf, M. S. (2011). The mechanisms linking health literacy to behavior and health status. American Journal of Health Behavior, 35 (1), pp. 118-128.

Östberg, A. L.; Skeie, M. S.; Skaare, A. B. \& Espelid, I.(2016). Caries increment in young children in Skaraborg, Sweden: associations with parental sociodemography, health habits, and attitudes. International Journal of Paediatric Dentistry, 27, pp. 47-55. Doi: 10.1111/ipd.12225.

Peñaranda, F. (2002). La educación a padres en los programas de salud desde una 
perspectiva de desarrollo humano. Revista Latinoamericana de Ciencias Sociales, Niñez y Juventud, 1 (1), pp. 207-230.

Ramfjord, S. P.; Morrison, E. C.; Burgett, F. G.; Nissle, R. R.; Shick, R. A.; Zann, G. J. \& Knowles, J. W. (1982). Oral Hygiene and Maintenance of Periodontal Support. Journal of periodontology, 53 (1), pp. 2630. Doi: 10.1902/jop.1982.53.1.26.

Schroder, K. E. E.; Ollis, C. L. \& Davies, S. (2013). Habitual Self-Control: A Brief Measure of Persistent Goal Pursuit. European Journal of Personality, 27 (1), pp. 82-95.

Secretaría de Salud y Asistencia (2011). Perfil Epidemiológico de la Salud Bucal en México 2010. México, D. F.: Inave, DGE, Salud.

Soria-Hernández, M.; Molina, N. \& Rodríguez, R. (2008). Hábitos de higiene bucal y su influencia sobre la frecuencia de caries dental. Acta Pediátrica de México, 29 (1), pp. 21-24.

Soto-Ugalde, A.; Sexto-Delgado, N. \& GontánQuintana, N. (2014). Intervención educativa en salud bucal en niños y maestros. Medisur, 12 (1), pp. 3 13. Recuperado de: http://medisur.sld.cu/index.php/medisur/ article/view/2571

Verplanken, B. \& Orbell, S. (2003). Reflections on Past Behavior: A Self-Report Index of Habit Strength1. Journal of Applied Social Psychology, 33 (6), pp. 1313-1330. Doi:10.1111/j.1559-1816.2003.tb01951.x.

Williams, N. J.; Whittle, J. G. \& Gatrell, A. C. (2002). The relationship between sociodemographic characteristics and dental health knowledge and attitudes of parents with young children. British dental journal, 193 (11), pp. 651-654. Doi: 10.1038/ sj.bdj.4801652.

Zimmerman, B. (2000). Self-Efficacy: An Essential Motive to Learn. Contemporary Educational Psychology, 25 (1), pp. 82-91. Doi:10.1006/ceps.1999.1016. 